\title{
La vinculación comunitaria en la educación intercultural, una estrategia pedagógica
}

\section{Community linkage in intercultural education, a pedagogical} strategy

Este trabajo sistematiza la práctica docente, investigación y vinculación, de instituciones que plantean la educación como factor de desarrollo. Su objetivo es mostrar la vinculación comunitaria como estrategia pedagógica que tiene al proyecto de investigación como eje articulador de los aprendizajes. Es un ejercicio de reflexión pedagógica y teórica, sobre la experiencia docente intercultural, que recupera la experiencia docente lograda entre 1994 y 2005 en dos instituciones de educación superior. En cuanto a los resultados, la colaboración resulta preponderante en el desarrollo de competencias, fortalece procesos organizativos y solidarios. En la enseñanza-aprendizaje los alumnos estudian su realidad para transformarla, con la participación comunitaria. En las conclusiones encontramos que es primordial continuar innovando sobre el diálogo del conocimiento científico y los saberes tradicionales; mantener la actualización epistemológica, metodológica, filosófica y política del plan de estudios; la formación continua de docentes para superar su práctica pedagogía tradicional.

Palabras clave: Interculturalidad; investigación; competencias; vinculación; mediación.

\footnotetext{
a Doctor. Director General, Alternativas para el Desarrollo Autogestionario, A. C., México. 凶elopezlob@hotmail.com
} 
This work systematizes the teaching practice, research and links, institutions that raise education as a factor of development. Its objective is to show the community bonding as a pedagogical strategy that has the research project as the articulating axis of the learning. It is an exercise of pedagogical and theoretical reflection on the intercultural teaching experience, which recovers the teaching experience achieved between 1994 and 2005 in two institutions of higher education. In terms of results, collaboration is predominant in the development of skills, strengthens organizational processes and solidarity. In teaching-learning students study their reality to transform it, with community participation. In the conclusions we find that it is essential to continue innovating on the dialogue of scientific knowledge and traditional knowledge; maintain the epistemological, methodological, philosophical and political updating of the curriculum; the continuous training of teachers to overcome their traditional pedagogy practice.

Key words: Interculturality; research; competences; linkage; mediation.

\title{
La vinculación comunitaria en la educación intercultural, una estrategia pedagógica
}

\author{
Community linkage in intercultural education, a pedagogical strategy
}

\section{Ubicación teórico-metodológica}

I presente trabajo se ubica en el marco de la teoría de la modificabilidad estructuEral cognitiva. Postula que "el organismo humano está dotado de la plasticidad y flexibilidad que le llevan a un alto grado de modificabilidad y lo hacen impredecible en su comportamiento y desarrollo" (Feuerstein, 1997, p. 11). Reconoce que la modificabilidad disminuye en las personas que no reciben una adecuada mediación, o cuando son condicionadas "por la herencia, la condición genética, la anomalía cromosómica, la privación o el medio ambiente” (p.13), sin embargo, esto se puede revertir al ofrecer condiciones y una intervención apropiada. 
Cuando esto sucede incrementa la inteligencia, es decir, "la propensión o tendencia del organismo a ser modificado en su propia estructura, como respuesta a la necesidad de adaptarse a nuevos estímulos, sean de origen interno o externo" (Feuerstein, 1997, p. 11). Implica el establecimiento de una relación dialéctica para la producción de conocimiento, con el apoyo de un sujeto más capaz, superando la transmisión de conocimientos y el papel pasivo del sujeto.

La mediación filtra, organiza, adecua y gradúa los estímulos externos para facilitar la construcción del conocimiento, considerando la zona de desarrollo próximo del sujeto mediado, es decir, considera "la distancia entre el nivel real de desarrollo, determinado por la capacidad de resolver independientemente un problema, y el nivel de desarrollo potencial, determinado a través de la resolución de un problema bajo la guía de un adulto o en colaboración con otro compañero más capaz" (Vygotsky, 1979, p. 133).

Esto requiere de un proceso dialéctico para la construcción del conocimiento, como parte de la mediación que se realiza a partir de la teoría denominada Experiencia del Aprendizaje Mediado (EAM), que "se produce por la interpretación de un ser humano iniciado e intencionado que media entre el mundo y el organismo, creando en el individuo la propensión o tendencia al cambio por la interacción directa con los estímulos" (Feuerstein, 1997, p. 15).

Esta teoría postula que hay dos modalidades que afectan la estructura cognitiva y emocional del sujeto cuando se exponen a estímulos internos y hechos del mundo, que permite la modificabilidad a través de una correcta mediación, siempre que se mantienen cuatro elementos indispensables, la intencionalidad, el significado, la trascendencia y el sentimiento de competencia.

Estos aportes teóricos se conjugan con la perspectiva teórica del constructivismo, que plantea la existencia de "una interacción entre el docente y los estudiantes, un intercambio dialéctico entre los conocimientos del docente y los del estudiante, de tal forma que se pueda llegar a una síntesis productiva para ambos y, en consecuencia, que los contenidos son revisados para lograr un aprendizaje significativo" (Ortiz, 2015, p. 94).

Esta perspectiva es la base del proceso de enseñanza-aprendizaje considerando que "el ser humano es activo constructor de su realidad, pero lo hace siempre en interacción con otros” (Ortiz, 2015, p. 95), en el que con una mediación pedagógica adecuada desarrolla habilidades cognitivas y prácticas, así como contenidos académicos, con una disposición a aprender a aprender. 
Esta perspectiva tiene sus bases en la teoría de Piaget, de Ausubel y de Vygotsky, que aportan elementos importantes del constructivismo. La teoría cognitiva de Piaget es conocida como evolutiva al postular etapas del desarrollo cognitivo que se vuelven más complejas conforme madura la persona. El aprendizaje significativo de Ausubel postula que "el sujeto relaciona las ideas nuevas que recibe con aquellas que ya tenía previamente, de cuya combinación surge una significación única y personal. Este proceso se realiza mediante la combinación de tres aspectos esenciales: lógicos, cognitivos y afectivos (Lamata \& Domínguez, 2003, como se citó en Ortiz, 2015, p. 98). La teoría de Vygotsky, "el aprendizaje social" sostiene que el aprendizaje es el resultado de la interacción del individuo con el medio (Ortiz, 2015), y que se logra en lo que denominó la zona del desarrollo próximo.

Estos aportes son la base para establecer una mediación en el proceso de enseñanza-aprendizaje, donde el estudiante incrementa su modificabilidad cognitiva, lo que les permite comprender conceptos más complejos o abstractos, modificar su realidad y adaptarse a nuevos contextos, como manifestación de su inteligencia.

En términos metodológicos, el presente trabajo se basa en la propuesta de la Investigación Acción Participativa (IAP), que tiene sus orígenes en los trabajos de Kurt Lewin quien "partía de la teoría psicosocial y proponía combinar teoría y práctica en la investigación-acción a través del análisis del contexto, la categorización de prioridades y la evaluación" (Balcazar, 2003, p. 60). Estos aportes fueron retomados en una perspectiva epistemológica de la IAP, donde plantea que "la experiencia le permite a los participantes 'aprender a aprender'. Este es un rompimiento con modelos tradicionales de enseñanza en los cuales los individuos desempeñan un papel pasivo y simplemente acumulan la información que el instructor les ofrece” (Balcazar, 2003, p. 61).

Plantea que es la propia comunidad, o miembros de ésta, quienes deben participar de manera activa en la investigación junto a los investigadores, en un proceso que crea conciencia de sí, de su mundo, de sus problemas y sus soluciones. En este sentido, es una acción que reivindica el papel de sujeto como tal y supera su condición de objeto de estudio; abre la posibilidad de que comprendan y narren su realidad, pero sobre todo que sean capaces de transformarla, de subvertir lo que no vale la pena vivir.

Es en este marco teórico-metodológico en el que se basa el presente trabajo, que retoma la experiencia educativa de instituciones de nivel superior que apuestan por una educación no convencional, para formar profesionales vinculados a sus comunidades, que promueven modos de vida buena. 


\section{El trabajo colaborativo y el proyecto de investigación}

Algunas instituciones como el Centro de Estudios para el Desarrollo Rural y la Universidad Veracruzana Intercultural se han creado con el propósito de impulsar modelos de educación alternativos que rompen esquemas tradicionales, donde el estudiante mantiene un papel pasivo, que limita su potencial, cuyo "único margen de acción que se ofrece a los educandos es el de recibir los depósitos, guardarlos y archivarlos" (Freire, 1972, p. 46). Por el contrario, impulsan procesos de enseñanza-aprendizaje vinculados a la comunidad, bajo una estrategia que se puede denominar "generación de situaciones educativas", que consiste en "situaciones que parten de la vida real, que permiten construir de manera sistemática conocimientos sobre esta realidad y que permiten aplicar estos conocimientos para transformar la situación estudiada" (Cesder, 1998, p. 44).

Los contenidos de las asignaturas se organizan y abordan en función de las necesidades conceptuales, teóricas y metodológicas que requieren en el proceso de investigación. En algunos momentos se hace necesario el apoyo del docente, quien facilita y gradúa los contenidos y los estímulos, de tal forma que crea las condiciones adecuadas para la construcción del conocimiento a través de mediaciones pedagógicas.

Algunas de estas experiencias son las que desarrolla la UVI, así como el CESDER, que tienen en común el propósito de vincular la formación académica con la intervención comunitaria, de tal forma que los estudiantes generan conocimientos a partir del reconocimiento y resignificación de la cultura, en relación con los actores sociales de las comunidades. Estudiar y a la vez intervenir en los procesos de producción agropecuarios; en la organización social; en la representación y ejercicio del poder desde el derecho consuetudinario y positivo; en los idiomas indígenas; en sus tradiciones y en las formas de sanación.

Estas instituciones educativas promueven la construcción del conocimiento desde el enfoque constructivista. Plantean que el aprendizaje se logra mediante "la incorporación de los datos a esquemas que poseen información organizada en patrones, así como funciones cognoscitivas que operan la información nueva y la ya adquirida” (Gómez, 200o, p. 40). Esto posibilita el desarrollo de estructuras mentales más complejas y con ello le permite al estudiante acceder a conocimientos complejos y abstractos, a resolver problemas de la vida cotidiana y "actuar ética e inteligentemente [...] [definido] como el uso de los procesos de pensamiento racional para llegar a decisiones, tomando en cuenta el bienestar de los involucrados" (Marzano, $1997 \mathrm{com}$ se citó en Gómez, 2000, p. 39). 
El interés por aprender, por implicarse en el proceso de enseñanza aprendizaje, "estriba no sólo en la adquisición de los conceptos y principios científicos, sino en la comprensión crítica de sus aplicaciones sociales y cotidianas implícitas” (Díaz, 2006, p.5). Una de las estrategias implementadas es el trabajo colaborativo en torno al desarrollo de un proyecto de investigación, donde los estudiantes trabajan bajo un mismo objetivo y con una adecuada mediación, articulan sus esfuerzos, se apoyan y "refuerzan los lazos afectivos que evitan las descalificaciones en virtud del color de la piel o la procedencia" (Milan, 2007, como se citó en Escarbajal, 2014, p. 38).

Cuando los estudiantes trabajan en equipo algunos se desempeñan como mediadores del conocimiento de aquellos compañeros que requieren ayuda. Es un espacio en el que ponen a disposición las capacidades de cada uno para lograr el éxito en la tarea. Desarrollan la socialización, alteridad, respeto, tolerancia, escucha, actitud propositiva, crítica y analítica. Potencia "el aprendizaje, al permitir la confrontación de puntos de vista y opiniones, ayuda a revalorizar la perspectiva propia y facilita el intercambio con el otro" (Maldonado, 2007, p. 275).

Lo que facilita la comprensión y trascendencia de los conocimientos es el sentido de utilidad, su aplicabilidad, por ello resulta estratégico pensar en proyectos donde se planteen resultados concretos en el corto plazo, ya que esto permite poner en práctica lo aprendido en el salón de clase, con lo cual los procesos de enseñanza-aprendizaje de las asignaturas se vuelven más interesantes.

Evitar escindir la investigación de los procesos de enseñanza-aprendizaje resulta estratégico. Representa para los estudiantes una oportunidad de "indagación y el manejo de problemas que no tienen soluciones inmediatas” (Gómez, 2000, p. 42), como las que se pueden obtener en una sesión de clase; de ahí que el proyecto les posibilita la puesta en práctica de los conocimientos adquiridos y el desarrollo del trabajo cooperativo, donde los estudiantes indagan, confrontan sus ideas y las argumentan frente a sus compañeros, para establecer conclusiones de forma colectiva.

En la enseñanza-aprendizaje basada en el trabajo colaborativo mediante el proyecto de investigación, que se plantea como eje articulador de los aprendizajes y el espacio de interacción social con objetivos y metas claras, los estudiantes "comparten, comparan y discuten ideas. Es a través de este proceso altamente interactivo que el alumno construye su propio conocimiento" (Cenich \& Santos, 2005, p. 9). De ahí el éxito de la propuesta pedagógica de estas instituciones, que proponen una pedagogía que parte del nivel de desarrollo cognitivo, del contexto, así también, del programa de contenidos para lograr las competencias y el compromiso de transformar la realidad estudiada. 


\section{La vinculación comunitaria: una estrategia pedagógica}

La educación intercultural no es privativa de los indígenas, aun cuando algunas instituciones educativas fueron creadas en las regiones con mayor presencia indígena. Es necesaria en todos los niveles educativos, dado que favorece la inclusión, la equidad, el reconocimiento de la diversidad y enriquece los procesos de formación, se evita la discriminación positiva, la segregación que "ha sido motivo para propiciar una educación empobrecida en lugar de enriquecida [...]. Esta toma de postura explica por qué las universidades interculturales son interculturales y no indígenas" (Schmelkes, 2013, p. 183), con el reto permanente de evitar su alienación a la lógica de la "exclusión inclusiva: despojados de su forma de vida, los individuos, las poblaciones, devienen primero sujetos negados [...] y luego resultan recolocados desde el despojo de su humanidad, [...] inscritos en el ordenamiento normativo vigente" (Berlanga, 2012, p. 3), donde sólo tienen acceso a modos de vida que corresponden al orden normativo de la ideología hegemónica que se impone y ordena la sociedad en clases sociales.

Las universidades interculturales, como otras instituciones que brindan una educación alternativa, intentan superar esta exclusión y establecen condiciones para el respeto, el diálogo, el aprendizaje mutuo, que da sentido, pertinencia y trascendencia a las propuestas de formación, con participación activa de estudiantes, familias y comunidades con quienes desarrollan proyectos que tienen doble propósito; por un lado, formar jóvenes profesionales promotores de la justicia, la equidad y la democracia, y por otro, contribuir al impulso de procesos sociales dirigidos a erradicar la pobreza.

Estos valores se vuelven centrales en una educación con población pluricultural, donde lo intercultural cobra relevancia, implica el respeto, pero no aceptación, producto de la resignación, sino desde la alteridad, se entiende la importancia de la diferencia del otro, y de los otros, que no son "como yo", ni como "nosotros"; es necesario dar la palabra, es decir, escuchar. "La escucha”, elemento central del diálogo entre personas y culturas, lo que posibilita la interacción para construir juntos.

Estas instituciones educativas han generado confianza en la población, esto favorece el trabajo de reconocimiento y resignificación de la cultura local, mediante la mejora de su práctica docente y de investigación-acción-participativa, que tienen a la realidad próxima del estudiante como "el punto de partida para un aprendizaje significativo, situado en la comunidad” (Baronnet, 2009 como se citó en Dietz \& Mateos, 2013, p. 19). También da la posibilidad de aprender junto con los actores sociales, 
los médicos tradicionales, los agricultores, los artistas comunitarios, las autoridades, y las familias, con quienes se produce y devuelve a la comunidad el conocimiento sistematizado, producto de la docencia, investigación y vinculación. Este último es "el proceso más vivo de [sic] relación con la comunidad a través de procesos de transformación comunitaria” (Schmelkes, 2013, p. 185). Esto implica que las instituciones educativas desarrollen competencias interculturales, mismas que se pueden agrupar en categorías como:

habilidades comunicativas; tolerancia de la ambigüedad; empatía; abertura mental; flexibilidad; capacidad de saber enfocar una tarea o una relación; postura positiva hacia el aprendizaje; tolerancia hacia estilos y culturas diferentes; conocimiento cultural; capacidad de tener éxito en contextos diferentes. (Earley \& Ang, 2003, como se citó en Surian, 2012, p. 211)

"La competencia intercultural posibilita la adaptación social de las personas a contextos culturales no propios" (Aneas, 2005, p. 4), pero no como un acto de enajenación, sino a partir del entendimiento y respeto a lo ajeno y lo diverso que enriquece la percepción del mundo diverso.

Es necesario desarrollar competencias interculturales en el propio proceso de vinculación comunitaria y no necesariamente como prerrequisito, a través de la mediación pedagógica entendida como "acción intencional que usando los recursos pertinentes, produce los cambios necesarios para conseguir los fines que pretendemos" (Fuentes, 1995, p. 327) y que sirve de "puente que se tiende entre el conocimiento que se desea aprender y el interlocutor" (Guoron, 2009, p. 68), considerando las perspectivas y experiencias de vida de los estudiantes, para "promover actitudes positivas hacia la diversidad cultural, frente al racismo y xenofobia, sin disociar lo cognitivo, lo afectivo y lo conductual" (García \& Sales 1997, p. 319). Esto representa una apuesta ética y política de toda institución educativa en la construcción de una sociedad incluyente, respetuosa, democrática y equitativa.

La mediación pedagógica intercultural que se plantea desde la diversidad cultural presente en el salón de clases y toma en cuenta la subjetividad de los estudiantes, hace trascender el conocimiento que tiene implícita la mediación del contenido que se "logra vinculándolo con la vida cotidiana y con la cultura local, lo cual también le da relevancia y sentido al aprendizaje" (Guoron, 2009, p. 71). Estos elementos generan la necesidad de aprender, y cuando el estudiante "aprende interculturalmente se sorprende, se muestra curioso, explora, investiga, inventa...” (Demetrio \& Favaro, 2009, como se citó en Escarbajal, 2014, p. 35), situación que el docente acompaña para 
asegurar el éxito del proceso y mejorar el autoestima, que implica el desarrollo del sentimiento de competencia, es decir, la capacidad de creer en sí mismo y que le ayuda en el crecimiento intelectual.

Uno de los primeros obstáculos en la clase es el desinterés de los estudiantes, que el docente supera con alguna actividad generadora de la necesidad de aprender, de descubrir y por lo tanto de trabajar. Lo que sigue es mantener la mediación para lograr el aprendizaje significativo, que "comprende la adquisición de nuevos significados y a la inversa, estos son producto del aprendizaje significativo" (Ausubel, Novak \& Hanesian, 2014, p. 48). Lo cual requiere de material apropiado, la intencionalidad de que el estudiante aprehenda y construya conocimientos.

El estudiante se implica activa y emocionalmente en el trabajo cuando "la mediación cumple con tres requisitos; a) despertar en el sujeto el interés por la tarea en sí; b) discutir con el sujeto acerca de la importancia que tiene dicha tarea; c) explicarle la finalidad que se persigue con las actividades y con la aplicación de las mismas" (Sánchez, 1989, p. 37). El proyecto de investigación vinculada establece las condiciones para el desarrollo de aprendizajes significativos que se acompaña en campo y en el aula de clases.

La práctica docente contempla estrategias didácticas que implican a los estudiantes emocionalmente y los confronta con problemas que exigen aplicar conocimientos y experiencias previas mediadas de acuerdo con su capacidad de comprensión, que los lleva a niveles más altos de conocimiento, manteniendo en todo momento el interés por aprender, por descubrir y lograr el objetivo planteado con suficiente claridad, "ya que sin importar lo significativa que sea la actitud del alumno, ni el proceso ni el resultado del aprendizaje serán posiblemente significativos si la tarea de aprendizaje no lo es potencialmente, y si tampoco es relacionable, intencionada y sustancialmente, con su estructura cognoscitiva" (Ausubel et al., 2014, p. 48).

La experiencia ha demostrado que la significatividad del aprendizaje ha resultado de la vinculación comunitaria que realizan los estudiantes a través de la investigación e intervención, confrontándolos con problemas reales. Les exige una decodificación y codificación permanente de saberes tradicionales y del conocimiento científico para construir uno nuevo.

En este ejercicio de vinculación comunitaria para lograr la significatividad en la mediación pedagógica, se reconoce y resignifica la realidad, se constata que coexisten varios mundos de vida compuestos por las relaciones de trabajo, las relaciones familiares, las interrelaciones sociales, la cosmogonía, costumbres y tradiciones, lo cual 
representa una oportunidad de enriquecerlos, mediante un entendimiento recíproco de las culturas, donde la escuela se convierte en un espacio compartido y de convivencias entre los estudiantes, docentes y actores sociales. Donde el convivir es el compartir las formas distintas de vivir, de aprender, de traducción mutua y de comprenderse, como posibilidad de construir juntos; entonces "la educación no debería de ser un instrumento para aprender a interpretar a los demás sino que tendría que ser una escuela de traductores, en la cual nos traducimos mutuamente" (Fornet-Betancourt, 2004, p. 51).

Esta práctica educativa fortalece la autoafirmación, autoestima, identidad cultural y se revalora la cultura, entendida como "la vida vivida, (...) el 'uso' o 'habla' de una versión particular del código universal de lo humano” (Echeverría, 1998, p. 161); que en su estudio se establece un proceso socio-cognitivo que abre posibilidades de comprenderla y enriquecerla, con procedimientos flexibles, que evitan "prácticas escolares que mantienen, acentúan y legitiman las desigualdades sociales de los estudiantes en base de no reconocer y valorar sus diferencias culturales" (Aguado, 2003, p. 4).

Es imperante la implementación de estrategias didácticas donde el docente es un mediador/facilitador del aprendizaje, que atiende a la "compleja imbricación de diferencias culturales, de clase social, de género, edad, nivel económico y creencias, que las considera más como constructos dinámicos, relaciones construidas socialmente, que características estáticas, fijas, adquiridas o inherentes a los grupos e individuos" (Aguado, 2003, p. 3), lo cual les demanda un trabajo permanente de ingenio, de creatividad y compromiso para estar innovando en la práctica docente.

Los procesos de aprendizaje en la educación intercultural parten del hecho de que "todas las culturas son valiosas, disfrutan el mismo estatus y validez, por tanto, todas tienen el mismo derecho a desarrollarse en plenitud" (García \& Sales, 2003, p. 19). Para que esto suceda es necesario que el Estado garantice las condiciones idóneas en términos de infraestructura y políticas, que faciliten el desarrollo de la docencia, investigación y vinculación con la sociedad.

\section{La investigación participativa en la educación intercultural}

La educación que ofertan las instituciones aquí consideradas, se caracteriza por su enfoque constructivista e intercultural, que mantiene una vinculación comunitaria aprovechando el conocimiento de los sabios locales y que articula sus acciones a par- 
tir de un proyecto de investigación. Algunos docentes han construido una ruta metodológica que considera una serie de fases e interfaces para revisar las acciones y resultados obtenidos, lo que permite corregir, complementar y preparar el desarrollo de la siguiente. Este proceso es acompañado en campo, así como en sesiones de tutoría, donde se reconocen los elementos conceptuales, metodológicos, heurísticos y axiológicos que requieren.

La investigación-acción-participativa (IAP) prevé la participación activa de los actores sociales no como informantes, sino como parte del equipo, que tiene intereses comunes sobre los que se toman acuerdos de colaboración. Esta es una "metodología implicativa” (Alberich, 2008), cuya perspectiva dialéctica plantea la necesidad de resarcir "las dos grandes rupturas del conocimiento científico [...]: sujeto cognoscenteobjeto cognoscible y análisis-intervención” (Palazón, 1993, p. 47), que propone no escindir la relación, por el contrario, hacerla visible y potenciarla, aprovechar el cúmulo de conocimientos milenarios construidos en las comunidades y crear las condiciones para que los propios sujetos los sistematicen y compartan, evitando así la interpretación de otros, de una cultura ajena y dominante.

Una vez que definen los intereses comunes y el alcance de la investigación participativa, "los actores sociales se convierten, en analista de su propia realidad" (Francés, Alaminos, Penalva \& Santacreu, 2015, p. 60), establecen acciones de corto, mediano y largo plazo, para lograr los objetivos planteados. Esto implica un proceso sistemático donde participan de forma activa en el diagnóstico, análisis y diseño de propuestas de intervención. Favorece la toma de conciencia de que otro mundo es posible y les permite construir conocimientos desde la praxis.

Desarrollar una pedagogía vinculada a las comunidades a partir del proyecto de investigación acción participativa desarrolla una cognición situada, la cual "es resultado de la actividad de la persona que aprende en interacción con otras personas en el marco de las prácticas sociales que promueve una comunidad determinada" (Díaz, 2006, p. 20), donde los conocimientos cobran sentido, por su utilización práctica, dispuestos para "atender las necesidades humanas y de la comunidad, mediante oportunidades educativas organizadas e intencionalmente estructuradas, que promueven aprendizaje y desarrollo, basados en la reflexión, reciprocidad, colaboración, y respeto a la diversidad" (Jacoby, 1996, como se citó en Díaz, 2006, p. 99).

Esta práctica pedagógica que privilegia el diálogo intercultural permite "interactuar de manera comprensiva y abierta al aprendizaje" (Díaz, 2013, p. 289), entre los estudiantes, docentes y actores sociales, quienes despliegan competencias intercul- 
turales como "un conjunto de habilidades cognitivas y características afectivas y de comportamiento que hacen desarrollar una afectiva interacción en una variedad de contextos culturales" (Bennett, 2008, como se citó en Escarbajal, 2014, p. 35), producto de una pedagogía dirigida a la producción de conocimientos, valores y actitudes para favorecer la interacción de los sujetos y comunidades en la construcción de proyectos colectivos que mejoren sus condiciones de vida.

\section{Conclusiones}

Se ha expuesto aquí de manera sucinta una serie de elementos que contribuyen a la reflexión para emprender una educación intercultural, con sentido, pertinencia y trascendencia, que considera la pluralidad de formas de aprender, ya que cuando se impone uno y sobre ese se evalúa, el problema no está en el estudiante que reprueba, sino en el método que se utiliza.

Las diversas formas de aprender: social, auditivo, kinestésico, visual, lógico e intrapersonal, se ponen en práctica cuando se enfrenta a los estudiantes a problemas reales, que les demanda poner en práctica sus distintas competencias. A la institución le demandan una adecuación y actualización permanente del currículo, así como elaborar un plan de desarrollo bajo un proceso participativo de las comunidades, donde la acciones contribuyen a mejorar las condiciones materiales y humanas de vida de las familias, producto de un trabajo sistemático y estratégico, de corto, mediano y largo plazo, que además de rescatar, preserve los saberes tradicionales, las lenguas originarias, las normas, modos de sanación, cosmovisión y la relación de respeto con la naturaleza.

No sólo la producción de conocimientos y habilidades es lo más importante. Son los valores como la sensibilidad, solidaridad, compromiso, arraigo, el trabajo cooperativo, el respeto, el diálogo y la democracia, que hacen una sociedad más justa, porque estos valores se disuelven, en la mayoría de las instituciones educativas. Estos valores se reafirman cuando se implican de manera activa en el proceso de formación vinculada a las comunidades para trabajar con las familias y actores sociales.

El aprendizaje significativo implica que el estudiante logra establecer conexión entre los conocimientos nuevos y los previos, para construir un pensamiento más complejo, que surge de una mediación del docente y de compañeros de clase, que ponen en práctica a partir del proyecto que se plantea como eje articulador de los 
aprendizajes, bajo la metodología investigación-acción-participativa. Esto permite el desarrollo de competencias intelectuales, de producción e interacción social.

Una formación profesional de estas características requiere docentes trabajadores, comprometidos y apasionados con la formación de nuevos profesionistas, que contribuyan en la construcción del buen vivir de las familias y comunidades. Es indispensable que el docente conozca el contexto desde donde se plantean los procesos de enseñanza-aprendizaje, los contenidos que despliega, así como el nivel medio del desarrollo cognitivo de los estudiantes, propiciando una participación protagónica de estos, además de hacerlos corresponsables en el proceso formativo.

Los docentes no escinden la educación de la investigación, hecho que propicia la participación activa de los estudiantes en el proceso para "desarrollar conocimiento, producir pensamiento y crear ciencia vinculada a los diferentes sectores, [...] que haga posible disminuir la brecha entre pobres y ricos" (Núñez, 2008, p. 9). Este es el reto de la educación, de hacer más prospera a la sociedad, que demanda la incidencia en las políticas públicas para construir mecanismos que permitan la distribución de la riqueza y que las familias hagan realidad sus propias aspiraciones de vida buena.

Si bien son las universidades interculturales a las que se les ha encomendado oficialmente por parte del Estado impartir una educación intercultural, existen instituciones que llevan una larga trayectoria en su impulso desde y con la población indígena y campesina, con sentido, pertinencia y trascendencia, comprometidos con la formación de niños, jóvenes y profesionales, con quienes construyen modos de vida dignos para sus familias, comunidades y regiones. Mantienen un plan de estudios vigente, siempre pertinente. Innovando es su forma de organizarse, de intervenir, de relacionarse y en las propuestas epistemológica, filosófica y política, como es el caso del CESDER y la Universidad Indígena campesina en Red.

En la educación intercultural no basta aceptar la existencia de otras culturas, si no se reconoce la importancia de su papel en la historia, de las prácticas ancestrales y del aporte al resto de la sociedad de elementos que son inspiradores para la realización de modos de vida dignos y de mejores relaciones con la naturaleza. Son los jóvenes formados profesionalmente quienes impulsarán estos procesos de cambio de manera creativa, crítica y propositiva, capaces de desenvolverse en diferentes contextos.

En el caso concreto de la UVI Totonacapan, algunos docentes revisan y estudian su didáctica apoyados en una ruta metodológica que organiza la práctica educativa en cuatro fases: 1) diagnóstico-indagar la realidad, explorar el medio; 2) análisis- 
planeación; 3) intervención comunitaria; y 4) sistematización de la experiencia. Esta propuesta tiene al proyecto de investigación como eje articulador de los aprendizajes que prevé interfaces en las que se revisa el logro de las metas, para retomarlas en la siguiente fase.

Lo que se pretende con la educación intercultural es construir conocimiento a la vez que se interviene en la realidad estudiada bajo un proceso planeado y sistemático, que contribuye a mejorar los modos de vida de las familias, donde el diálogo en el sentido más amplio mantiene la posibilidad de enriquecerse y de enriquecer a otras culturas. Es un asunto de todos y principalmente de las mayorías, quienes ven a las minorías diferentes. Implica también interacción, intercambio, ruptura del aislamiento, reciprocidad, solidaridad, así como reconocimiento y aceptación de otros saberes, cosmovisiones y prácticas ancestrales.

Estas experiencias educativas sobre las que hemos reflexionado tienen el proyecto como eje articulador de los conocimientos, la vinculación comunitaria, el enfoque pedagógico constructivista y el trabajo en equipo. Elementos comunes en el desarollo de una pedagogía que forma profesionalmente y para la vida. Se mantienen vigentes y pertinentes en su perspectiva, pedagógica, filosófica y política, a partir de la reflexión de su quehacer.

Hoy están planteando nuevos retos pedagógicos que articulan tres elementos que han sido centrales: la interculturalidad, el constructivismo y el aprendizaje dialógico, para ofrecer mejores oportunidades en la construcción del conocimiento a partir del aprovechamiento de la diversidad cultural y las inteligencias diversas que presentan los estudiantes, desde el enfoque de comunidad de aprendizaje, donde se privilegia el trabajo cooperativo, refuerzan los afectos y se evita la segregación, a la vez que se transforma el contexto en el que desarrollan la pedagogía basada en la participación activa de todos y que requiere actualización permanente del plan de estudios, innovación pedagógica, didáctica.

La UVI es vanguardista en su propuesta pedagógica, a pesar de los grandes retos que tiene ante sí: políticas administrativas y financieras rígidas que limitan el trabajo y la dificultad para sostenerse financieramente, mientras que otras como el CESDER, enfrentan la incertidumbre permanente de lograr los financiamientos de sus propuestas educativas, siempre innovadoras. 
Eloy López Lobato

\section{Lista de referencias}

Aguado, T. (2003). Pedagogía intercultural. España: McGraw-Hill/Interamericana de España.

Alberich, T. (2008). IAP, redes y mapas sociales: desde la investigación a la intervención social. Portularia, 8(1), 131-151. Recuperado de http://rabida.uhu.es/dspace/handle/10272/2178

Aneas, M. A. (2005). Competencia intercultural, concepto, efectos e implicaciones en el ejercicio de la ciudadanía. Revista Iberoamericana de Educación, 36(13), 1-9. Recuperado de https://rieoei.org/RIE/article/view/3004

Ausubel, D. P., Novak, J., \& Hanesian, H. (2014). Psicología Educativa, un punto de vista cognoscitivo. México: Trillas.

Balcazar, F. (2003). Investigación acción participativa (I AP): Aspectos conceptuales y dificultades de implementación. Fundamentos en Humanidades, 4(7-8), 59-77.

Berlanga, G. B. (2012). De otro modo la discriminación: acerca de la producción social de la vida campesina e indígena como vidas que no merecen la pena vivirse [mensaje en un blog]. Recuperado de http://www.ucired.org.mx/memorias/item/43produccion-social-de-la-vida-campesina-e-indigena/

Cenich, G., \& Santos G. (2005). Propuesta de aprendizaje basado en proyectos y trabajo colaborativo: experiencia de un curso en línea. Revista Electrónica de Investigación Educativa, 7(2), 1-18. Recuperado de http://redie.uabc.mx/vol7no2/contenido-cenich.html

Centro de Estudios para el Desarrollo Rural. (1998). Una educación para el medio rural: una propuesta pedagógica. México: Castillo.

Díaz, B. F. (2006). Enseñanza situada: Vínculo entre la escuela y la vida. México: McGraw-HiII/Interamericana.

Díaz, T. M. (2013). Etnografía de los saberes campesinos en procesos educativos. Los saberes interculturales de la educación agropecuaria a nivel medio superior. El estado de la cuestión. En S. Hernández, M. Ramírez, Y. Manjarrez \& A. Flores (Eds.), Educación intercultural a nivel superior: Reflexiones desde diversas realidades latinoamericanas (pp. 285-298). México: Poligráfica.

Dietz, G., \& Mateos, S. (2013). Una década de educación intercultural en México: Debates entre empoderamiento indígena y transversalización de la diversidad. En S. Hernández, M. Ramírez, Y. Manjarrez \& A. Flores, (Eds.), Educación intercultural a nivel superior: Reflexiones desde diversas realidades latinoameri- 
canas (pp. 11-33). México: Poligráfica.

Echeverría, B. (1998). La modernidad de lo barroco. México: Ediciones Era.

Escarbajal, F. A. (2014). La educación intercultural en los centros educativos. Revista Electrónica Interuniversitaria de Formación del Profesorado, 17(2), 29-43. doi:10.6018/reifop.17.2.197291

Feuerstein, R. (1997). La teoría de la modificabilidad cognitiva estructural. En L. Leberr, R. Garbo \& J. M. Martínez(Coords.), ¿Es modificable la inteligencia? (pp. 11-23). España: Bruño.

Fornet-Betancourt, R. (2004). Reflexiones de Raúl Fornet-Betancourt sobre el concepto de interculturalidad. México: Consorcio intercultural.

Francés, F., Alaminos, A., Penalva C., \& Santacreu, O. (2015). La investigación participativa: métodos y técnicas. Ecuador: Pydlos.

Freire, P. (1972). Pedagogía del oprimido. Argentina: Tierra Nueva/Siglo XXI.

Fuentes, N. (1995). La mediación y las teorías del desarrollo cognitivo. El profesor como mediador en el proceso de enseñanza aprendizaje. En M. D. Calero (Coord.), Modificación de la Inteligencia, sistemas de evaluación e intervención (pp. 340-365). España: Pirámides.

García, R., \& Sales, A. (1997). Educación intercultural y formación de actitudes. Programa pedagógico para desarrollar actitudes interculturales. Revista Española de Pedagogía, 55(207), 317-336. Recuperado de https://revistadepedagogia. org/wp-content/uploads/2007/06/207_rafaelagarcia.pdf

García, R., \& Sales, A. (2003). Programas de educación intercultural. España: Desclée De Brower.

Gómez, L. F. (2000). De la teoría general a la enseñanza en el aula. Sinéctica. Revista Electrónica de Educación, 17, 9-51. Recuperado de https://sinectica.iteso.mx/ index.php/sInectica/article/view/386

Guoron, P. (2009). Interculturalidad y mediación pedagógica intercultural. Guatemala: Ministerio de educación de Guatemala.

Maldonado, P.M. (2007). El trabajo colaborativo en el aula universitaria. Laurus, 13(23), 263-278. Recuperado de http://www.redalyc.org/articulo.oa?id=76102314

Núñez, J. (2008). La relación educación superior y el desarrollo local. En P. M. Sierra, (Coord.), Grupos dinámicos de información: Formación de actores en el desarrollo local (pp. 6-11). Cuba: Centro de Intercambio y Referencia Iniciativa Comunitaria-Memoria Popular Latinoamericana.

Ortiz, D. (julio-diciembre, 2015). El constructivismo como teoría y método de ense- 
ñanza. Sophia, Colección de Filosofía de la Educación, 19, 93-110. Recuperado de https://sophia.ups.edu.ec/index.php/sophia/article/view/19.2015.04

Sánchez, P. (1989). Modificabilidad cognitiva y P.E.I. Colección nueva escuela. España: Bruño.

Schmelkes, S. (2013). Las universidades interculturales en México: sus retos y sus necesidades actuales. En S. Hernández, M. Ramírez, Y. Manjarrez \& A. Flores, (Eds.), Educación intercultural a nivel superior: Reflexiones desde diversas realidades latinoamericanas (pp. 179-19o). México: Poligráfica.

Surian, A. (enero-abril, 2012). Aprendizaje y competencias interculturales. $\mathrm{Ra} \mathrm{Xi-}$ mhai, 8(2), 205-222. Recuperado de http://www.redalyc.org:9081/articulo. oa? id $=46123366009$

Vygotsky, L. (1979). El desarrollo de las funciones psicológicas superiores. Barcelona: Grijalbo. 